\title{
Dislocation Grain Boundary Interactions in Irradiated Metals
}

\author{
Ian M. ROBERTSON ${ }^{1}$, Bai CUI', Mo-Rigen $\mathrm{HE}^{1}$ \\ ${ }^{1}$ Dept. of Materials Science and Engineering, University of Wisconsin-Madison, (Madison, USA) \\ ${ }^{2}$ Dept. of Mechanical and Materials Engineering, University of Nebraska-Lincoln (Lincoln, USA)
}

The behavior of dislocations in unirradiated and ion-irradiated austenitic stainless steels has been observed directly by performing straining experiments in-situ in the transmission electron microscope. This has enabled direct observation of how dislocations interact with each other, with the irradiation produced obstacles and with grain boundaries. These experiments have provided insight as to the mechanisms by which dislocations annihilate the irradiation produced defects to form a channel as well as determination of the conditions that control slip transfer across a grain boundary and how it is impacted by the presence of the irradiation produced defects.

The mobility of dislocations across a grain are fundamentally different in unirradiated and irradiated austenitic stainless steels. In the unirradiated case, pre-existing as well as newly generated dislocations move rapidly, smoothly and as a continuous line elements through the grain. In contrast in irradiated metals, pre-existing dislocations or ones created prior to irradiation are rendered immobile by the irradiation. This locking of dislocations by the irradiation was demonstrated directly by straining an electron transparent sample in-situ in the TEM to generate mobile dislocations, irradiating the sample, continuing the straining, and observing the response of the dislocations [1]. The mobility of these dislocations was very limited. The deformation was carried by generating new dislocations from either grain boundary sources or small cracks generated during sample preparation. This observation demonstrates that pre-existing dislocations are not responsible for the formation of dislocation channels in deformed irradiated metals. A second difference is the movement of dislocations through the field of obstacles, it now occurs in an irregular, jerky and discontinuous manner. The initial dislocation velocity was low as the channel with reduced obstacle density was formed. The velocity increased with the passage of additional dislocations, but never become a simple easy glide path with dislocations moving as in the unirradiated material. Instead with the passage of additional dislocations cross-slip events against barriers invisible in the TEM became more numerous. This lead to the formation of long straight dislocations along the boundary between the irradiated matrix and the dislocation channel [2].

On intersecting a grain boundary dislocations can be accommodated within it. This occurred in both the unirradiated and irradiated materials. These accommodated dislocations may retain the matrix Burgers vector or dissociate into grain boundary type dislocations. These dislocations can be either sessile or glissile within the grain boundary. If the dislocations are glissile, the build-up of stress is distributed along the grain boundary and the location on the grain boundary from which dislocations are emitted is unlikely to be coincident with that of dislocation impact. Importantly, many dislocations were observed to be accommodated within the grain boundary and a pile-up of dislocations developed against it before slip was transmitted across it [3]. A consequence of this is that the dislocations emitted from the grain boundary source are in general not the same as the ones that intersected it. That is, new dislocations are created and emitted from within the grain boundary. The exception is for screw dislocations intersecting a twin boundary where the line of intersection of the two slip planes in the grain boundary is collinear with the line direction, Burgers vector, of the dislocation in both grains. The process of accommodating the incoming dislocations in and the emission of dislocations from the grain boundary disrupts the internal structure of the grain boundary and for high levels of transfer can cause a displacement of the grain boundary.

The propagation of dislocations through grain boundaries in unirradiated FCC metals has been shown to be governed by the magnitude of the Burgers vector of the grain boundary dislocation generated by the process of transmission of slip across the grain boundary $[3,4]$. The local resolved shear stress on the selected slip system must be sufficient to propagate the dislocations away from 
the grain boundary source into the grain. In irradiated FCC metals, the slip system activated to transmit slip is still governed by the magnitude of the grain boundary dislocation generated, but now the local resolved shear stress acting on the activated slip system must be of sufficient magnitude to propel the emitted dislocations through the irradiation hardened grain [5,6]. Evidence supporting this claim was found in the in-situ TEM experiments, as the magnitude of the local shear stress, through the addition of dislocations to the pile-up, had to be increased before the dislocations could propagate away from the grain boundary. Effectively, the irradiated matrix transitions the rate limiting step for slip transfer across a grain boundary from one of dislocation nucleation to dislocation propagation. If this local shear stress is insufficient the dislocations are not emitted from the grain boundary and slip is not transferred across it.

An interesting question to ask is what happens in situations in which the magnitude of the local resolved shear stress never becomes sufficient for slip transfer to occur. In such cases, one possibility is for the grain boundary to relieve the accumulated stress by disrupting the grain boundary structure by nucleating and propagating a crack along it. Although such events are rare they are possible and have been observed in both in-situ TEM straining experiments as well as in macroscale mechanical property tests. This observation has important implications for breaking the protective oxide and exposing the base metal to the environment, which has implications for irradiation assisted stress corrosion cracking. Examples of the different interactions dislocations can have with a grain boundary are illustrated in Figure 1, which shows dislocation accommodation in the grain boundary with no slip transmission, Fig. 1a; dislocation slip along the grain boundary, Fig. 1b; slip transmission involving one incoming system generating two outgoing systems, Fig. 1c; displacement of the grain boundary following extensive slip transmission, Fig. 1d, and crack nucleation and propagation along a grain boundary, Fig. 1e.
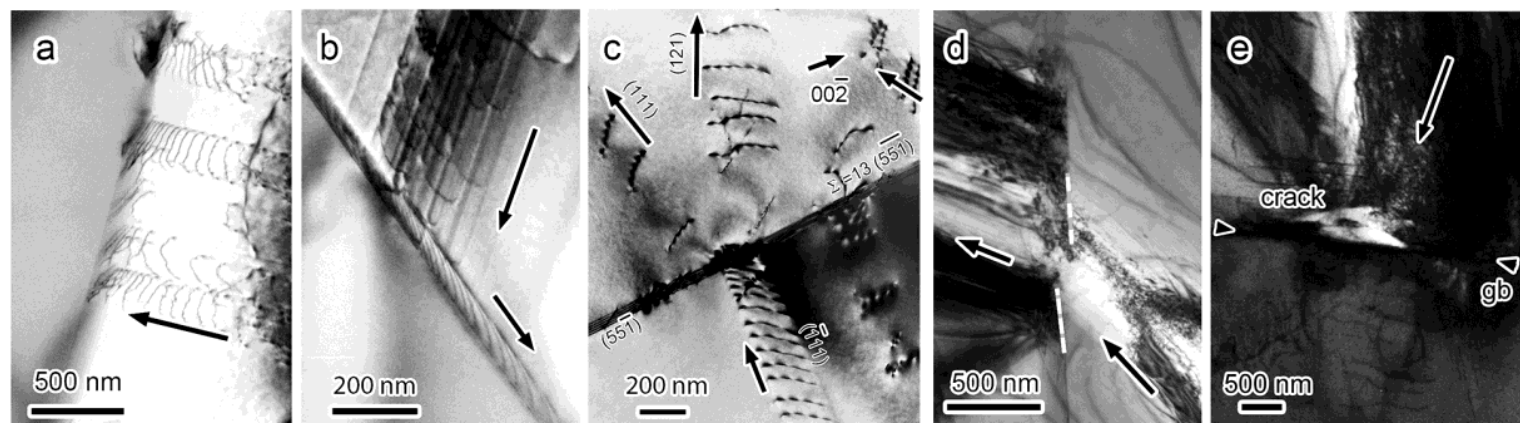

Fig. 1: Dislocation interactions with grain boundaries: a. accommodation, b. slip along grain boundary, c. slip transmission, d. slip transmission showing change in boundary structure and $e$. displacement of grain boundary due to slip transmission. a-d unirradiated and e. irradiated.

\section{References}

[1] Briceño M, Fenske J, Dadfarnia M, Sofronis P, Robertson IM. Effect of ion irradiation-produced defects on the mobility of dislocations in 304 stainless steel. J. Nucl. Mater. 2011; 409:18.

[2] Kacher J, Liu GS, Robertson IM. In situ and tomographic observations of defect free channel formation in ion irradiated stainless steels. Micron 2012; 43:1099.

[3] Kacher J, Eftink B, Cui B, Robertson IM. Dislocation interactions with grain boundary interactions. Current Opin. Solid State and Mater. Sci. 2014; 18227.

[4] Spearot DE, Sangid MD. Insights on slip transmission at grain boundaries from atomistic simulations. Current Opin. Solid State and Mater. Sci. 2014; 18:188.

[5] Cui B, McMurtrey MD, Was GS, Robertson IM. Micromechanistic origin of irradiation-assisted stress corrosion cracking. Phil. Mag. 2014:1.

[6] Cui B, Kacher J, McMurtrey M, Was G, Robertson IM. Influence of irradiation damage on slip transfer across grain boundaries. Acta Mater. 2014; 65:150.

Research supported by the U.S. Department of Energy, Office of Basic energy Sciences, Division of materials Sciences and engineering under Award DE-FG02-08ER46525. 


\title{
Dislocation Grain Boundary Interactions in Irradiated Metals
}

\author{
Ian M. Robertson ${ }^{1}$, Bai Cui², Mo-Rigen $\mathrm{He}^{1}$
}

'Dept. of Materials Science and Engineering, University of Wisconsin-Madison, (Madison, USA) ${ }^{2}$ Dept. of Mechanical and Materials Engineering, University of Nebraska-Lincoln (Lincoln, USA)

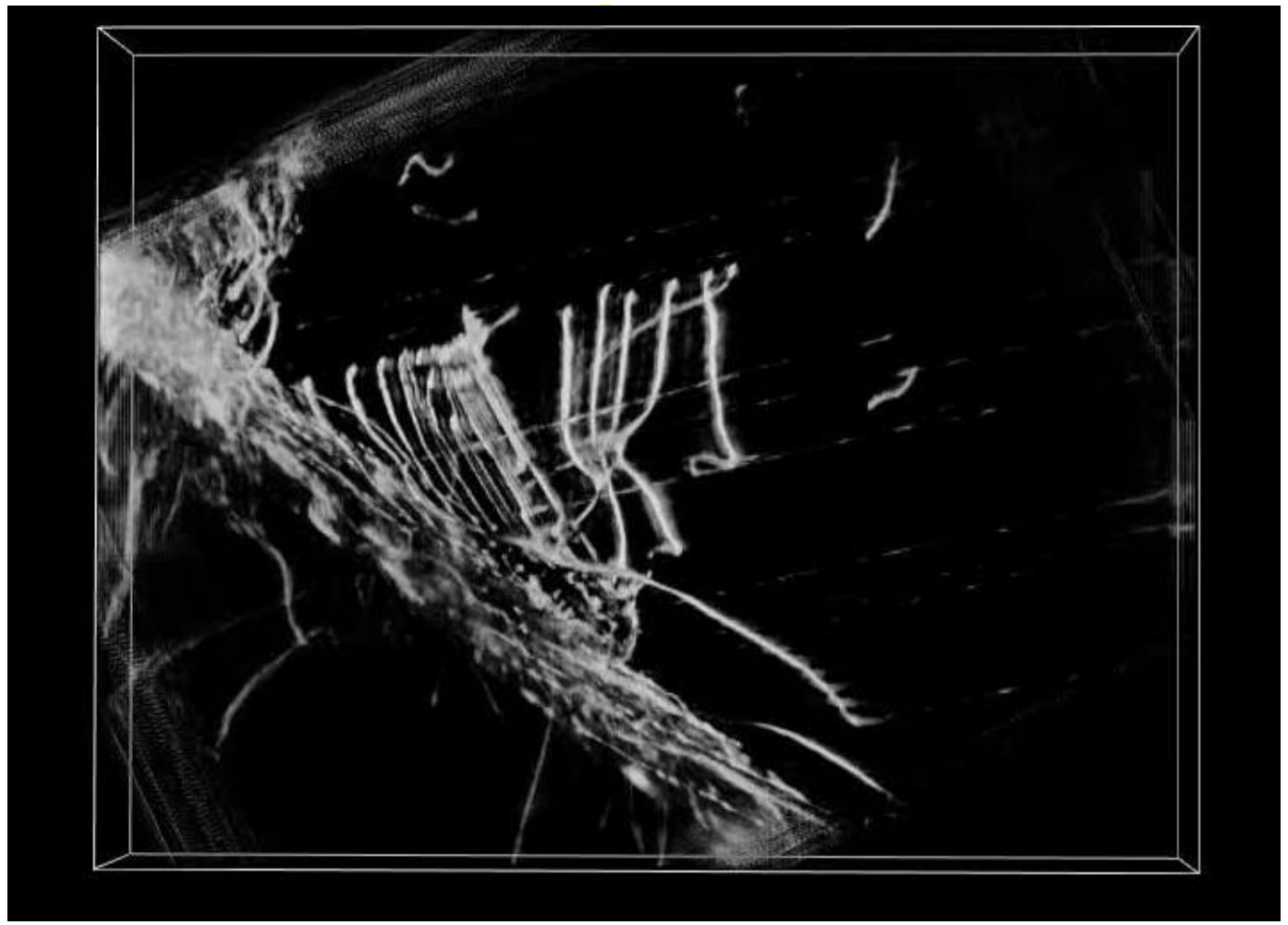




\section{Loading mechanism: sample forms used}

Conventional form

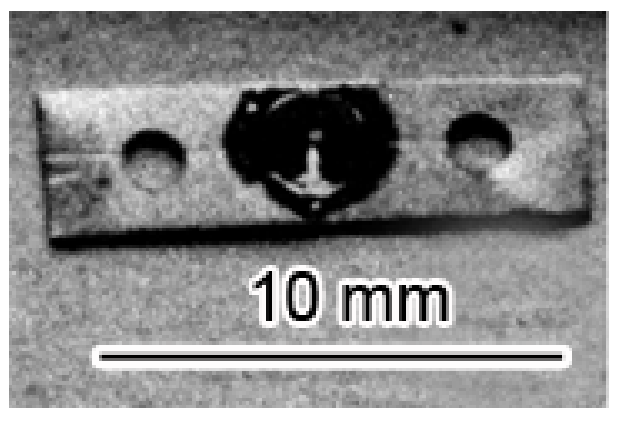

Sacrificial template with sample attached

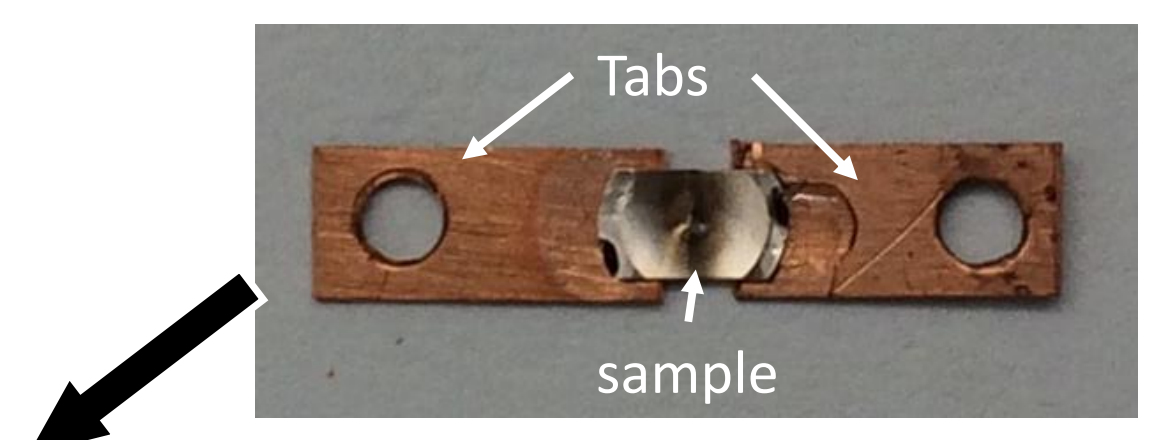

Focused Ion Beam machined sample: Control of loading direction with respect to interfaces
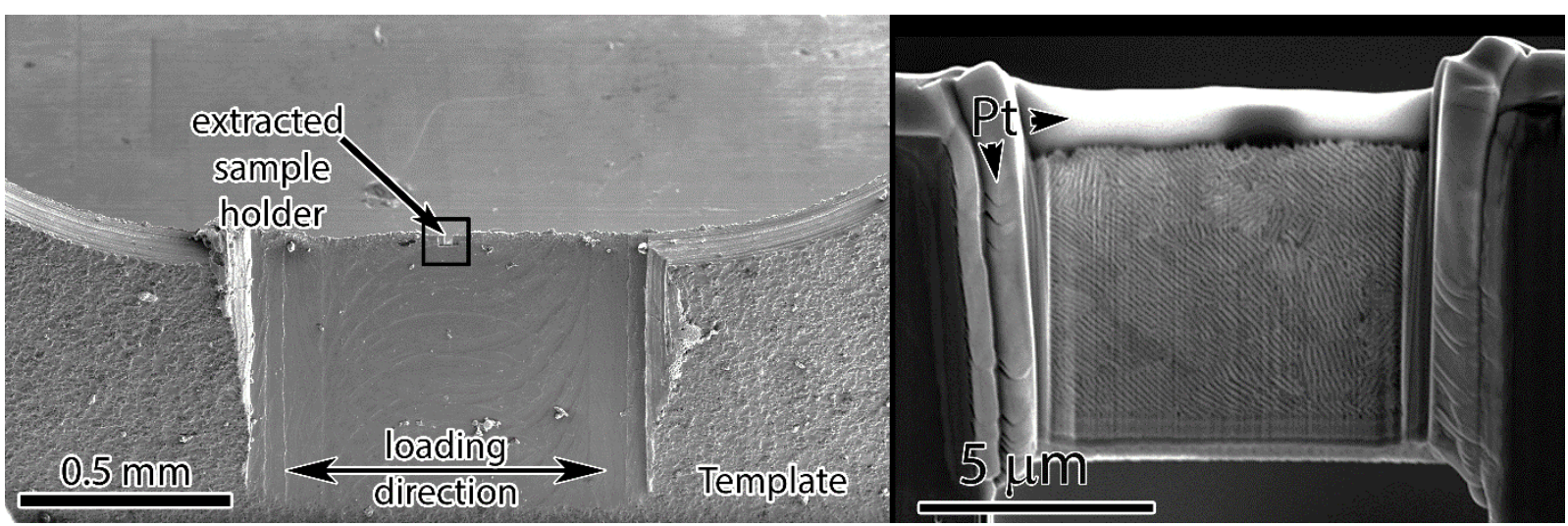

Field and Papin Ultramicroscopy. 2004 Dec;102(1):23-6.

Advantages:

Disadvantage: simple sample form, suitable for any material. temperature effects can be studied. no ability to measure directly the applied load / displacement or the strain. 


\section{Slip Transfer Criteria - FCC systems}

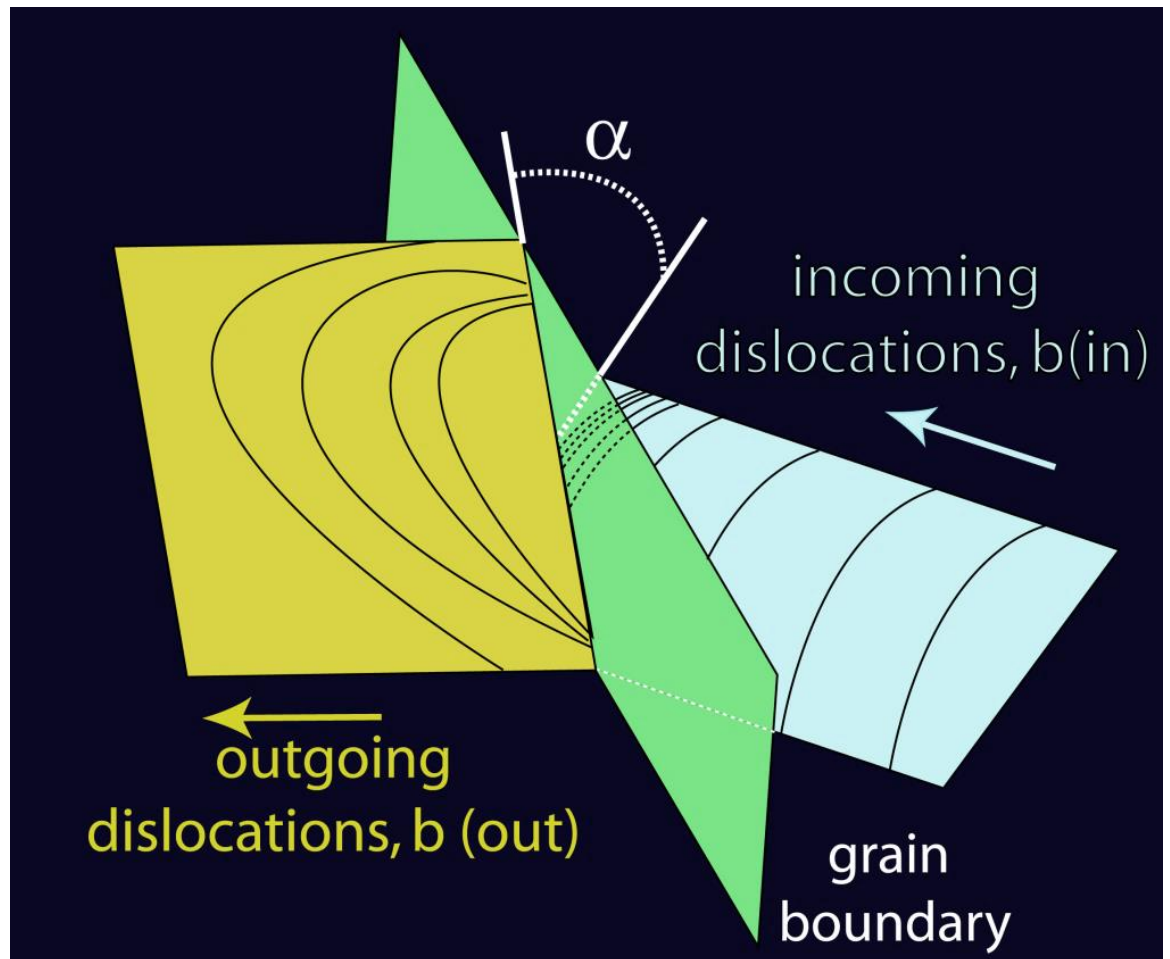

Slip Transfer Criteria - FCC systems

The magnitude of the Burgers vector of the residual dislocation generated in the grain boundary by the transfer process should be a minimum.

$$
b_{\text {in }}-b_{\text {out }}=b_{r}
$$




\section{Dislocation Interactions with a Grain Boundary}

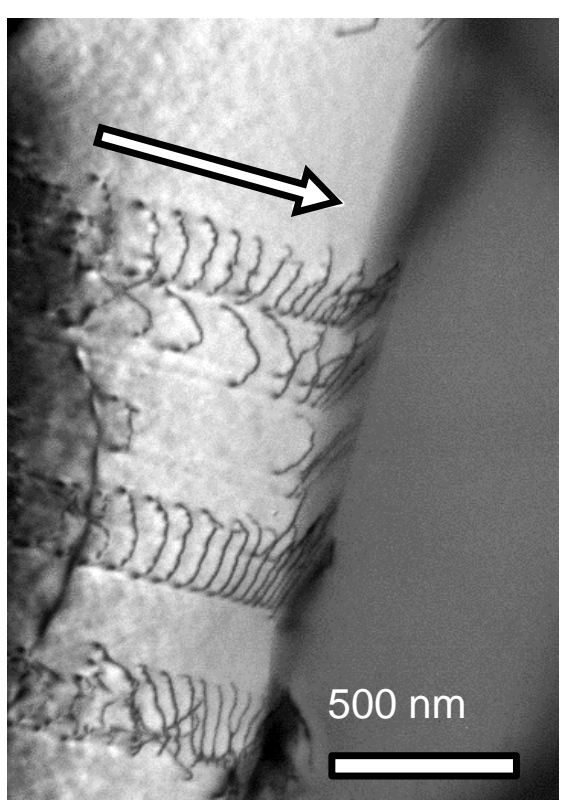

Dislocation accumulation in the grain boundary

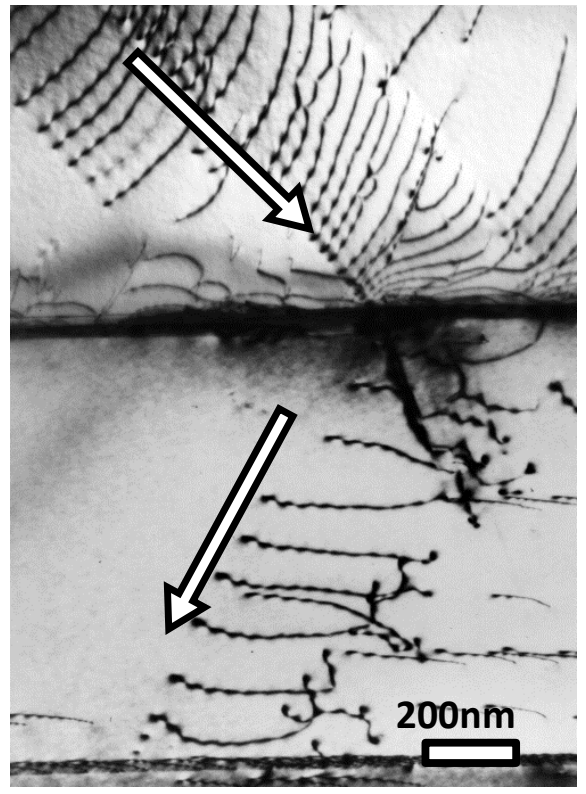

Slip transmission across the grain boundary

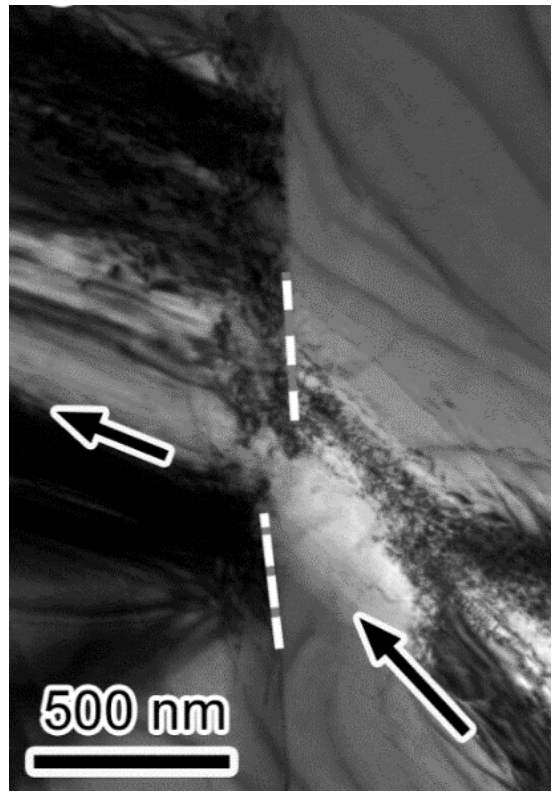

Disruption of the grain boundary by intense dislocation slip

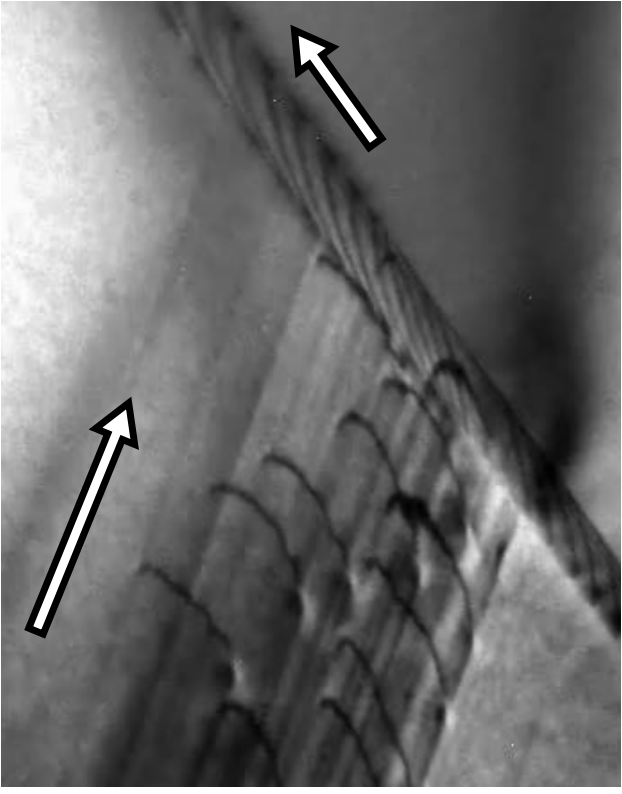

Accommodation and slip along the grain boundary

Findings:

- Dislocations can be accommodated in grain boundary with no slip transfer. Can retain matrix Burgers vector or become grain boundary dislocations. Disruption of grain boundary structure.

- Dislocations can be mobile in the grain boundary plane.

- $\quad$ Slip transfer results in the creation of a residual grain boundary dislocation, which can be considered as a change in strain energy density or even structure. More than one slip system can be activated.

- $\quad$ Transfer process can cause a rotation of a grain boundary. 


\section{Dislocation interactions with grain boundaries - unirradiated fcc}
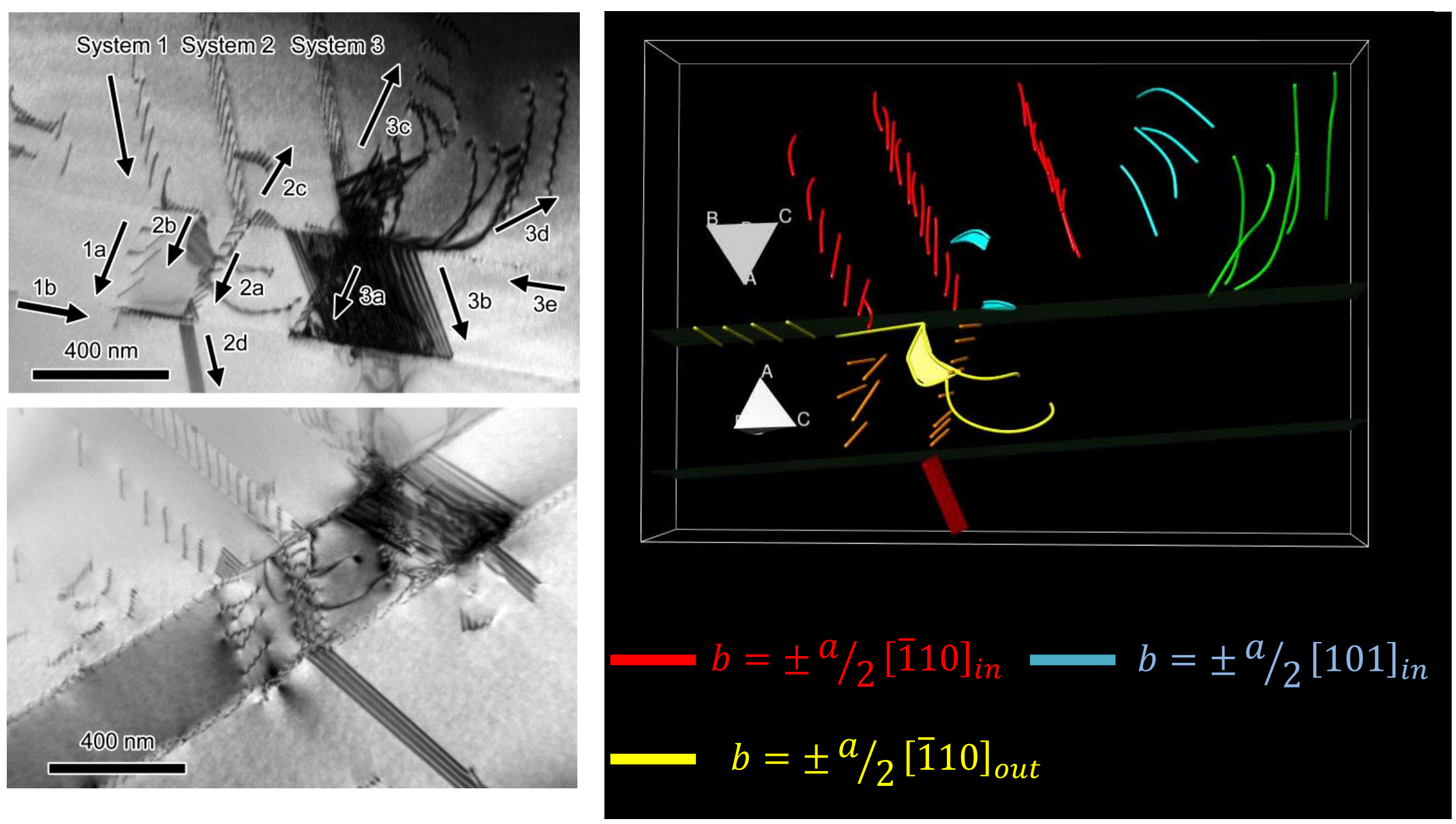


\section{Predicting the activated slip system}

Results for slip system 1
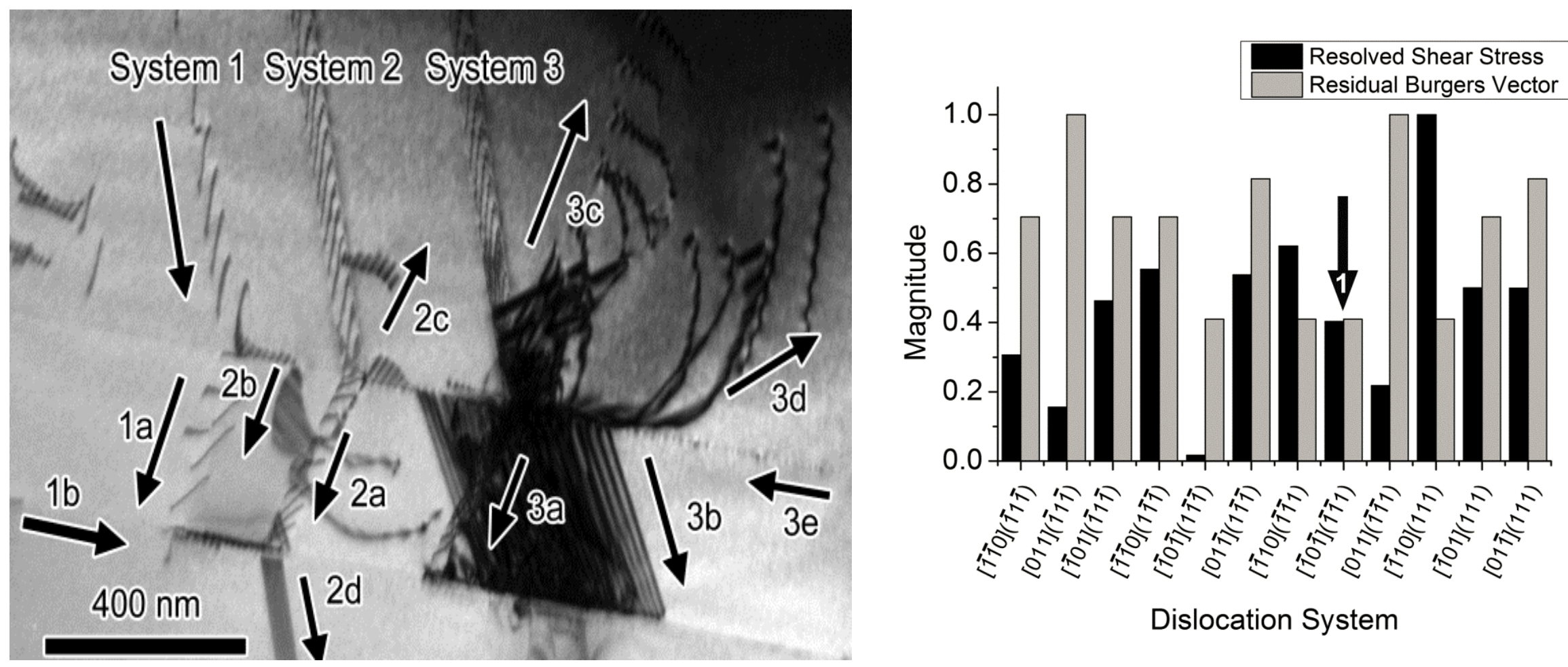

System activated in response to the impact of system 1 is determined by the magnitude of the Burgers vector of the dislocations left in the grain boundary following the transfer. The resolved shear stress acting on the system is low. 


\section{Grain boundary failure at the macroscale.}
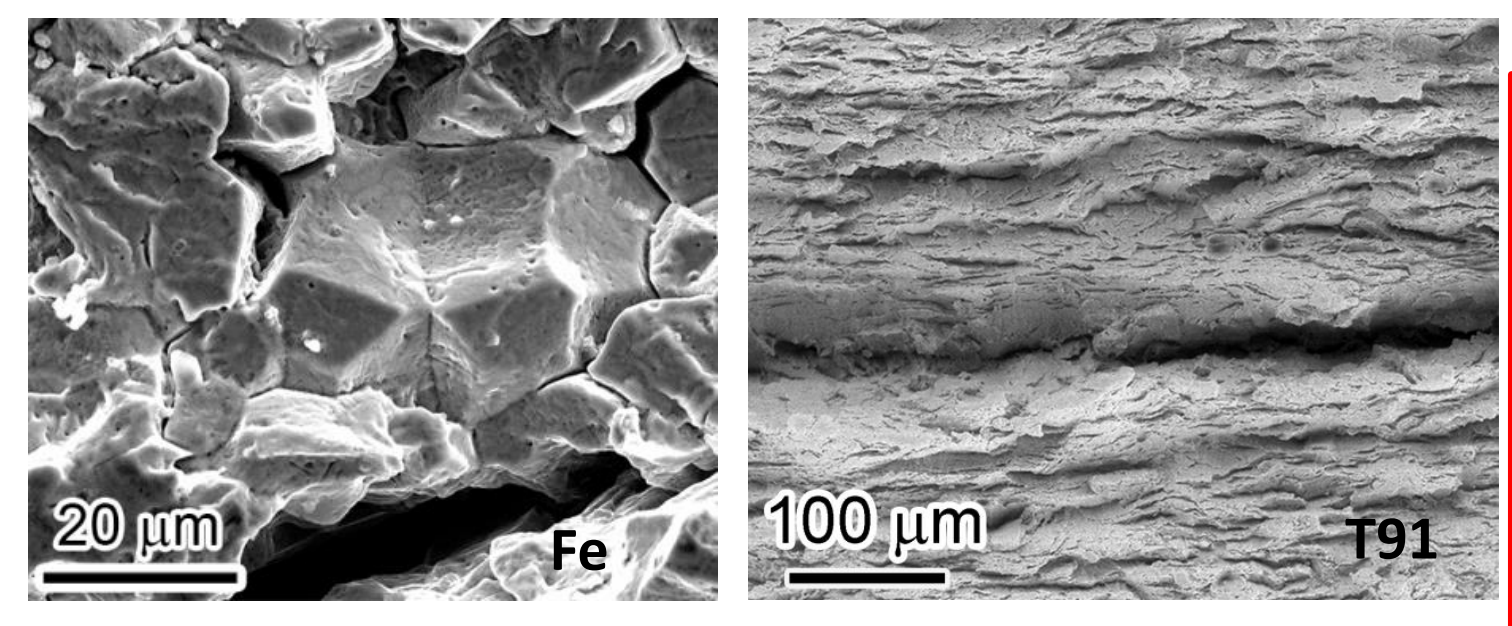

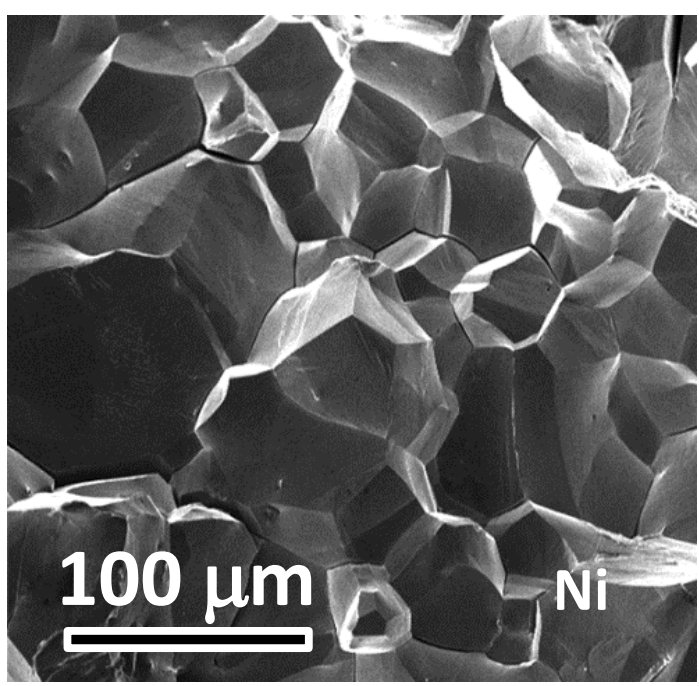

Hydrogen-induced intergranular failure

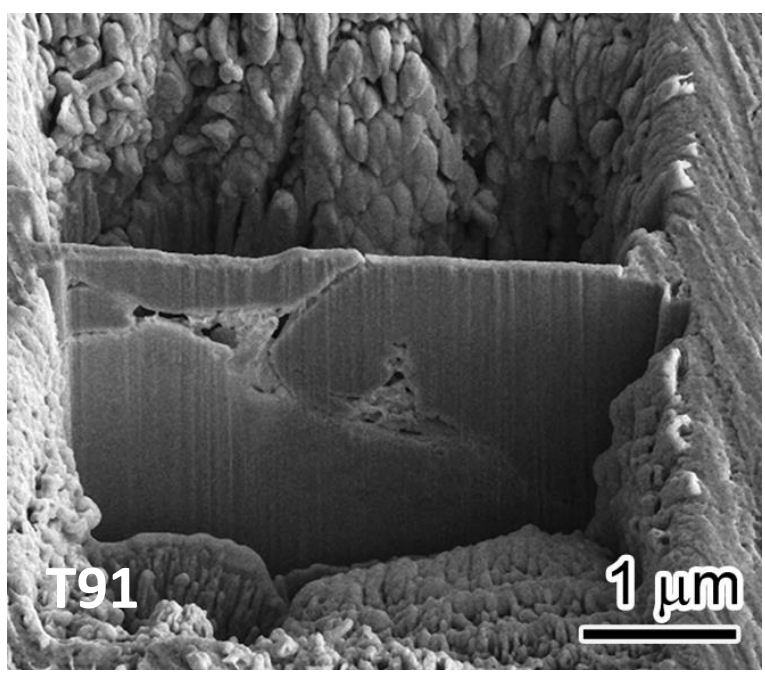

Liquid metal induced intergranular failure
Irradiation assisted intergranular stress corrosion cracking
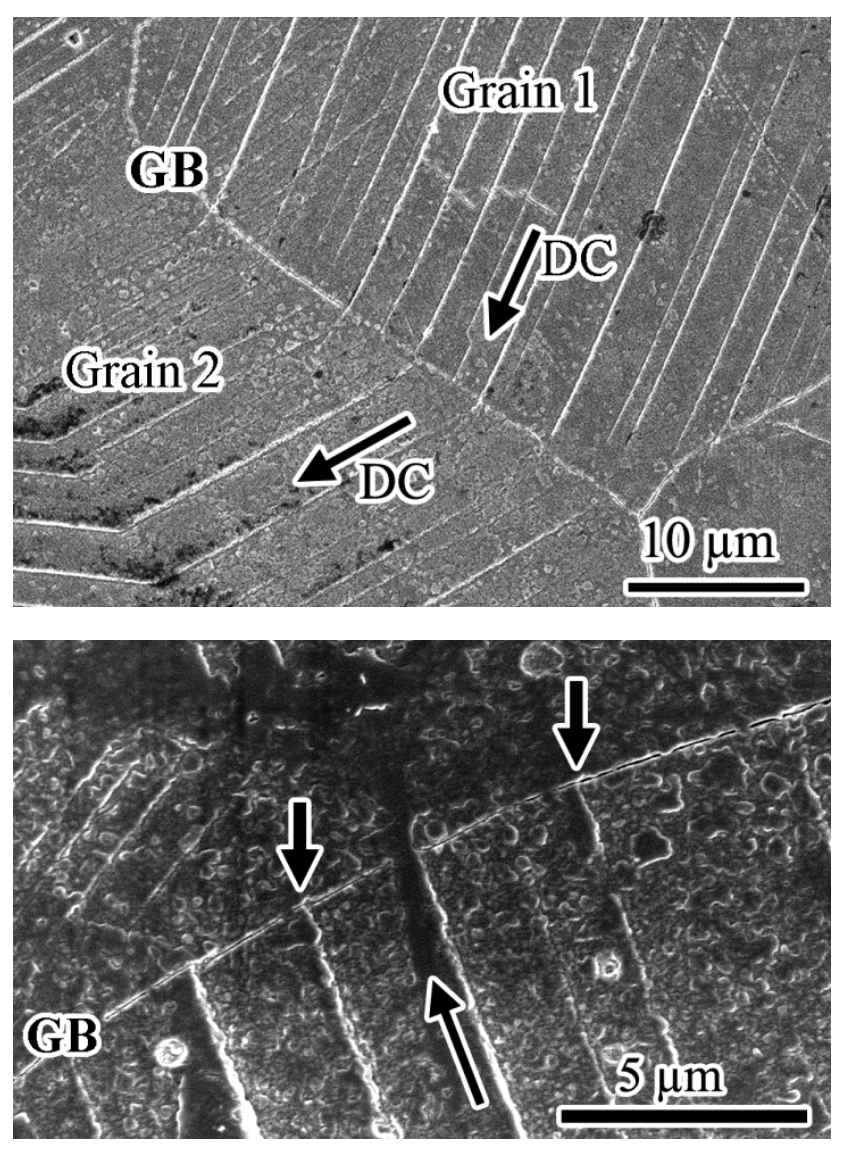

Stainless steel

Do plasticity process play any role in these intergranular failures? 


\section{Influence of ion irradiation on the mobility of pre-existing mobile dislocations}

Difference images
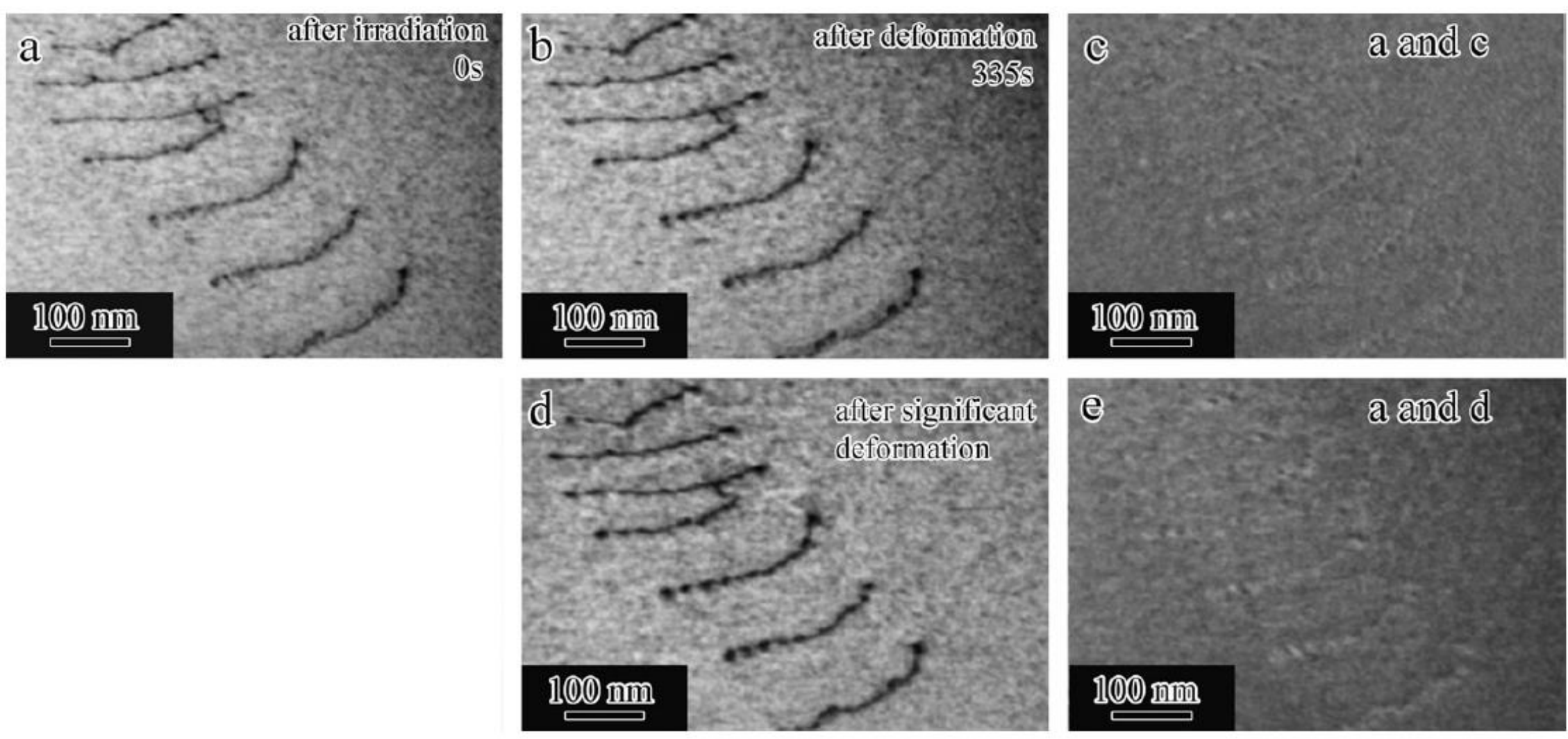

Pre-existing mobile dislocations rendered immobile by ion irradiation. 


\section{Comparing dislocation motion in irradiated and unirradiated metals}

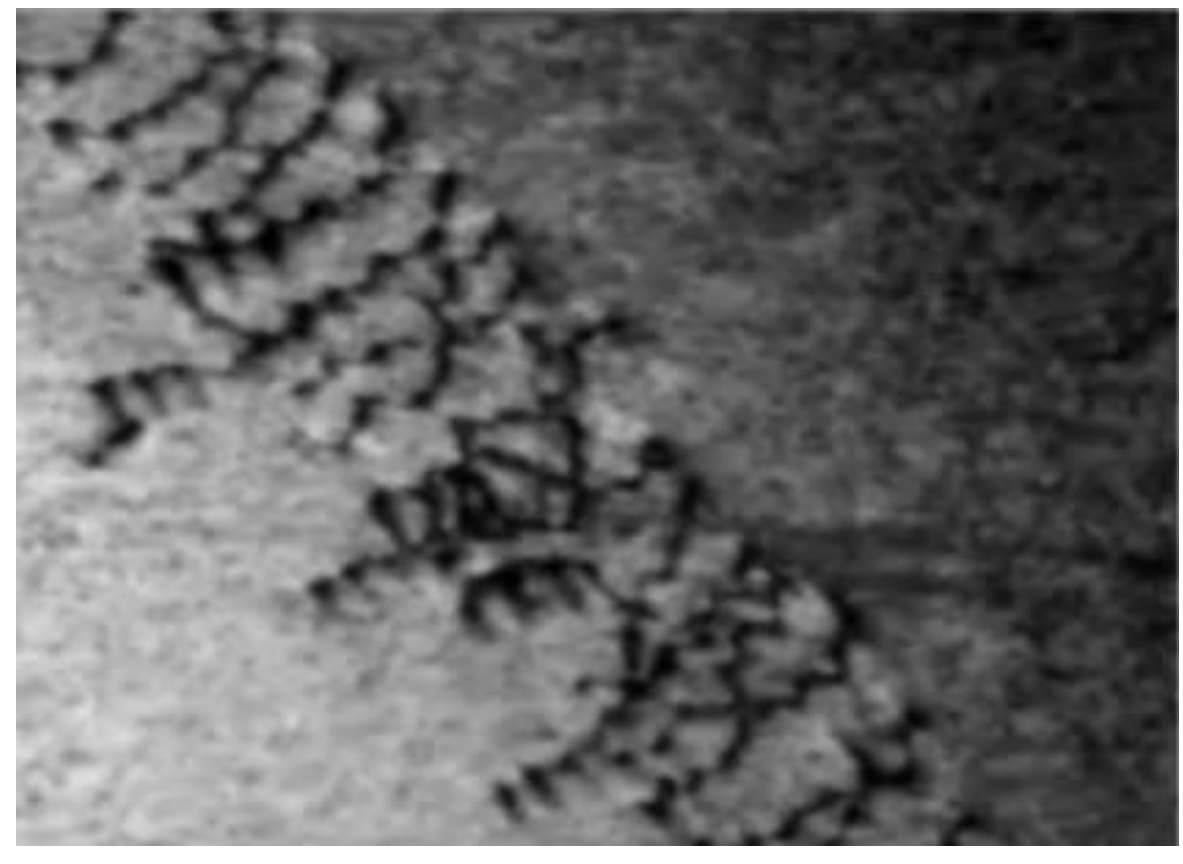

unirradiated

Irradiated - accelerated

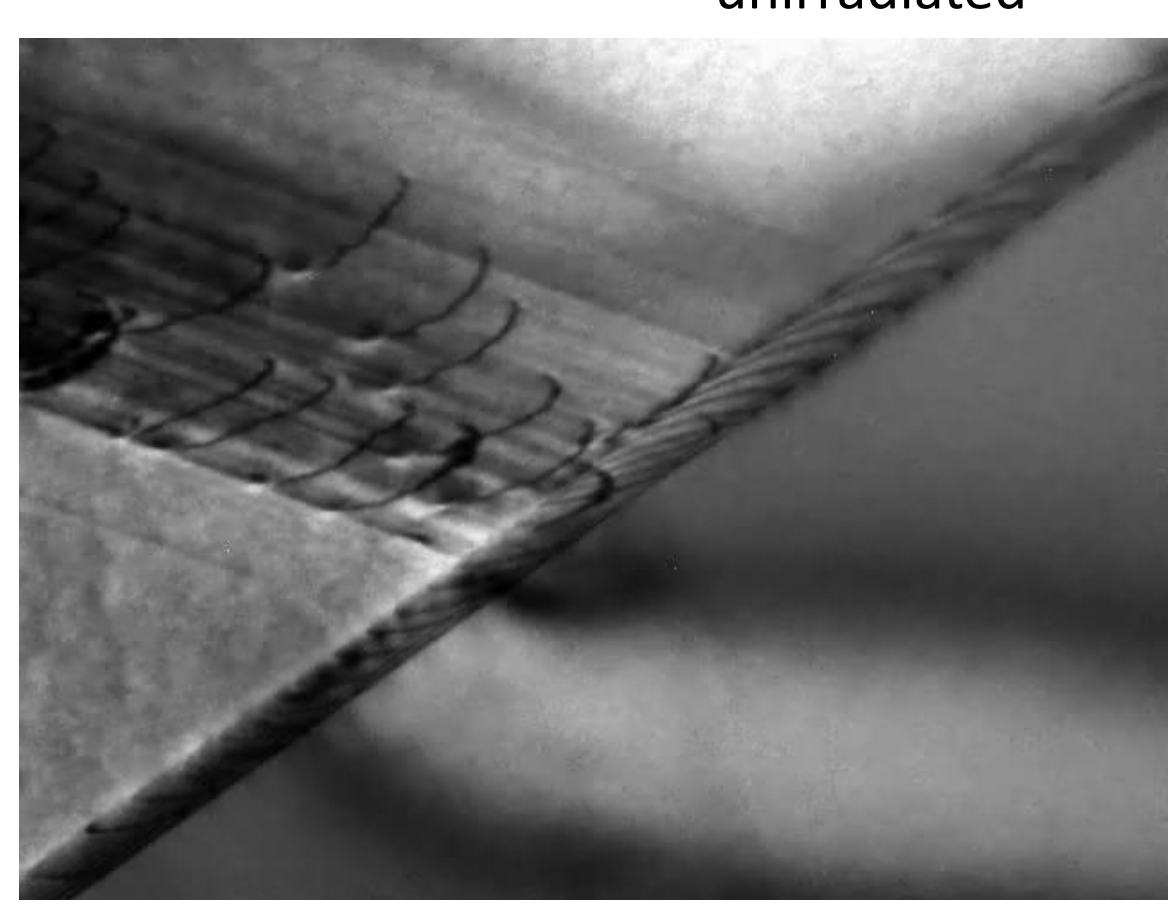

Dislocation processes sluggish, segmented and discontinuous due to the interactions with the field of irradiation produced defects. Dislocation velocity increases with continued loading. 


\section{Dislocation interactions with stacking-fault tetrahedra at elevated temperature.}

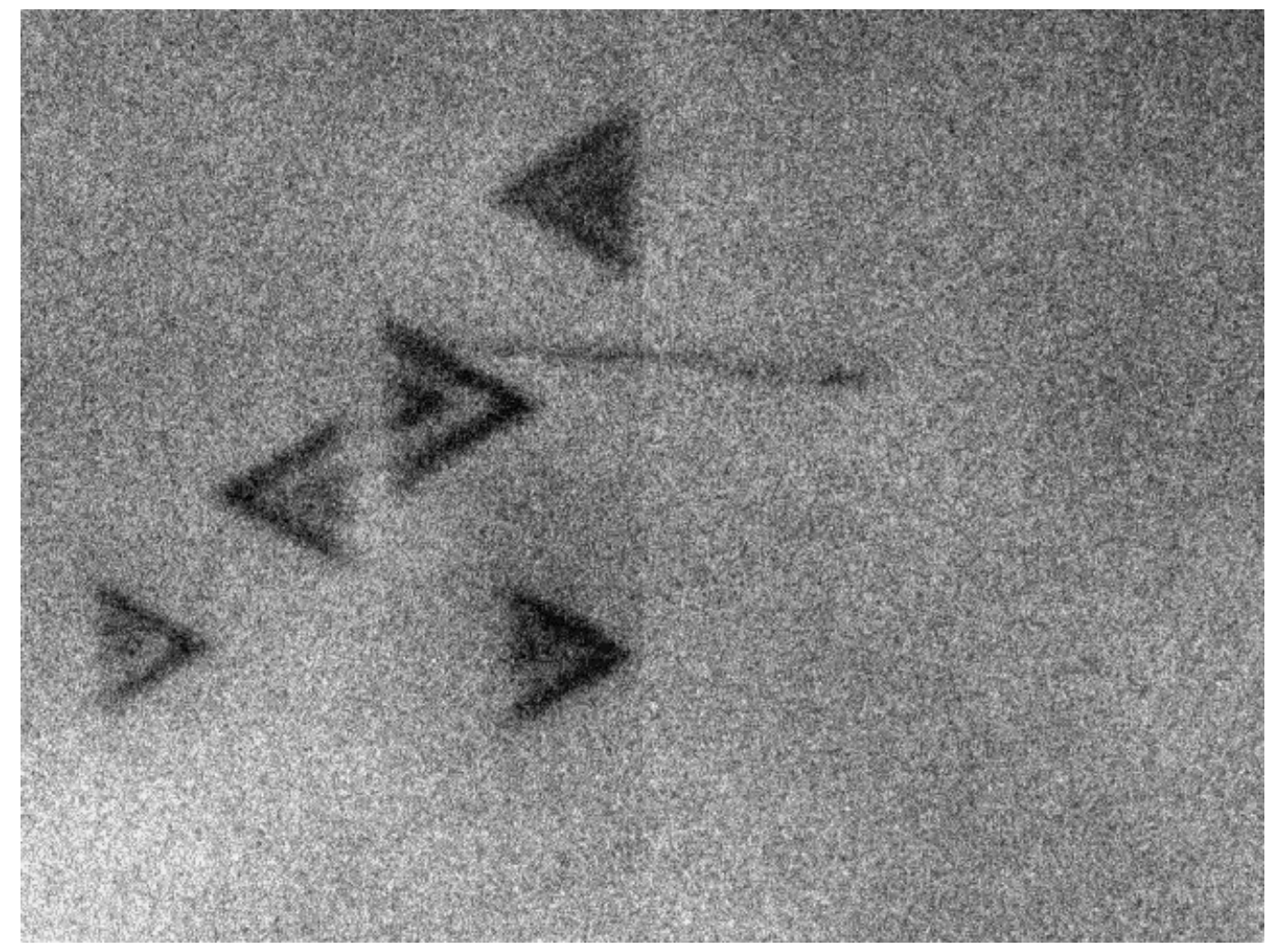

Identifying the reason for the different responses is hindered by the lack of spatial information. Need to introduce electron tomography to recover the information lost in the electron beam direction. 


\section{Dislocation interactions with stacking-fault tetrahedra at elevated temperature.}

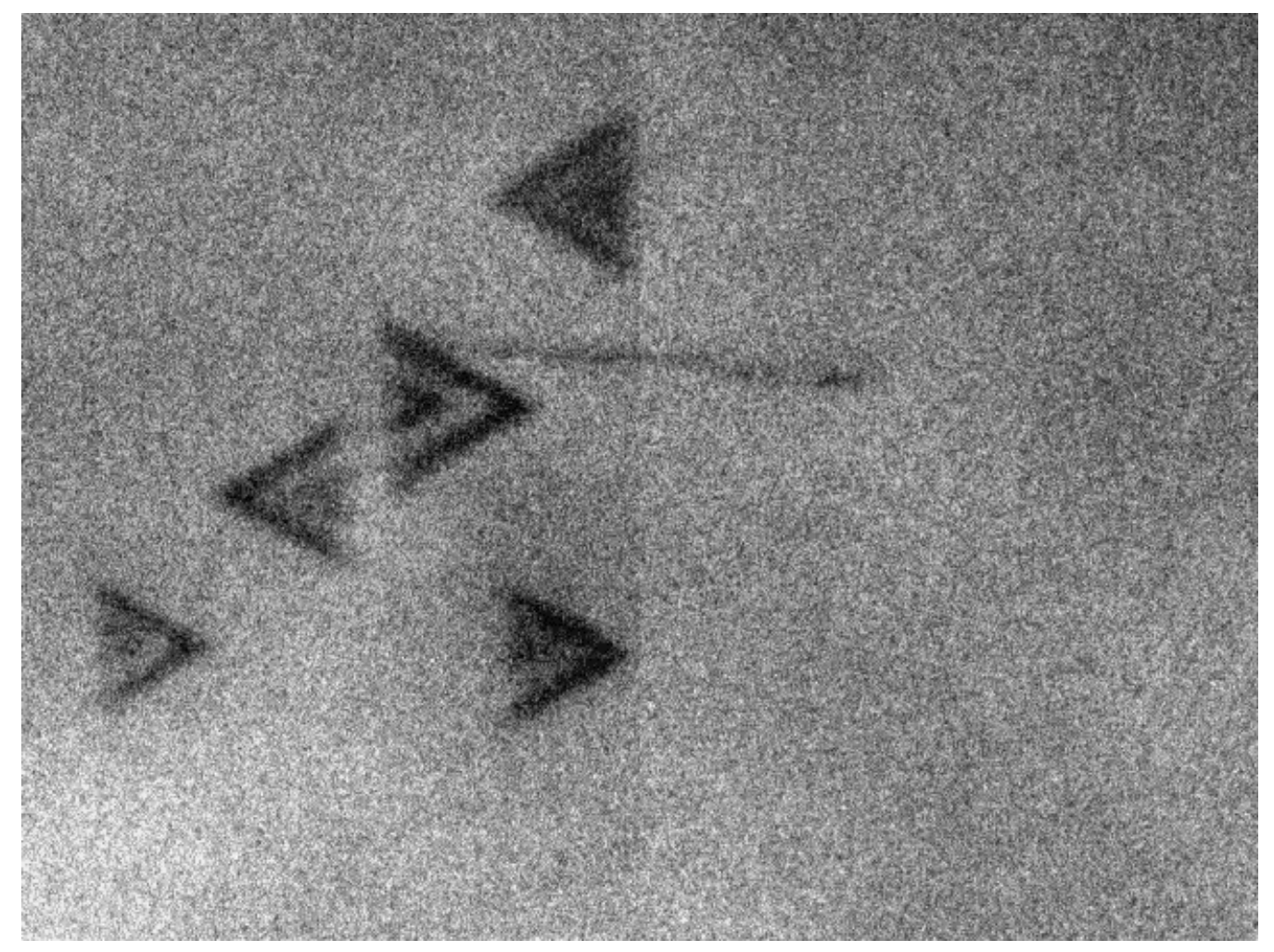

Identifying the reason for the different responses is hindered by the lack of spatial information. Need to introduce electron tomography to recover the information lost in the electron beam direction. 


\section{Interactions with individual defects - Stacking-fault tetrahedra. Gold}

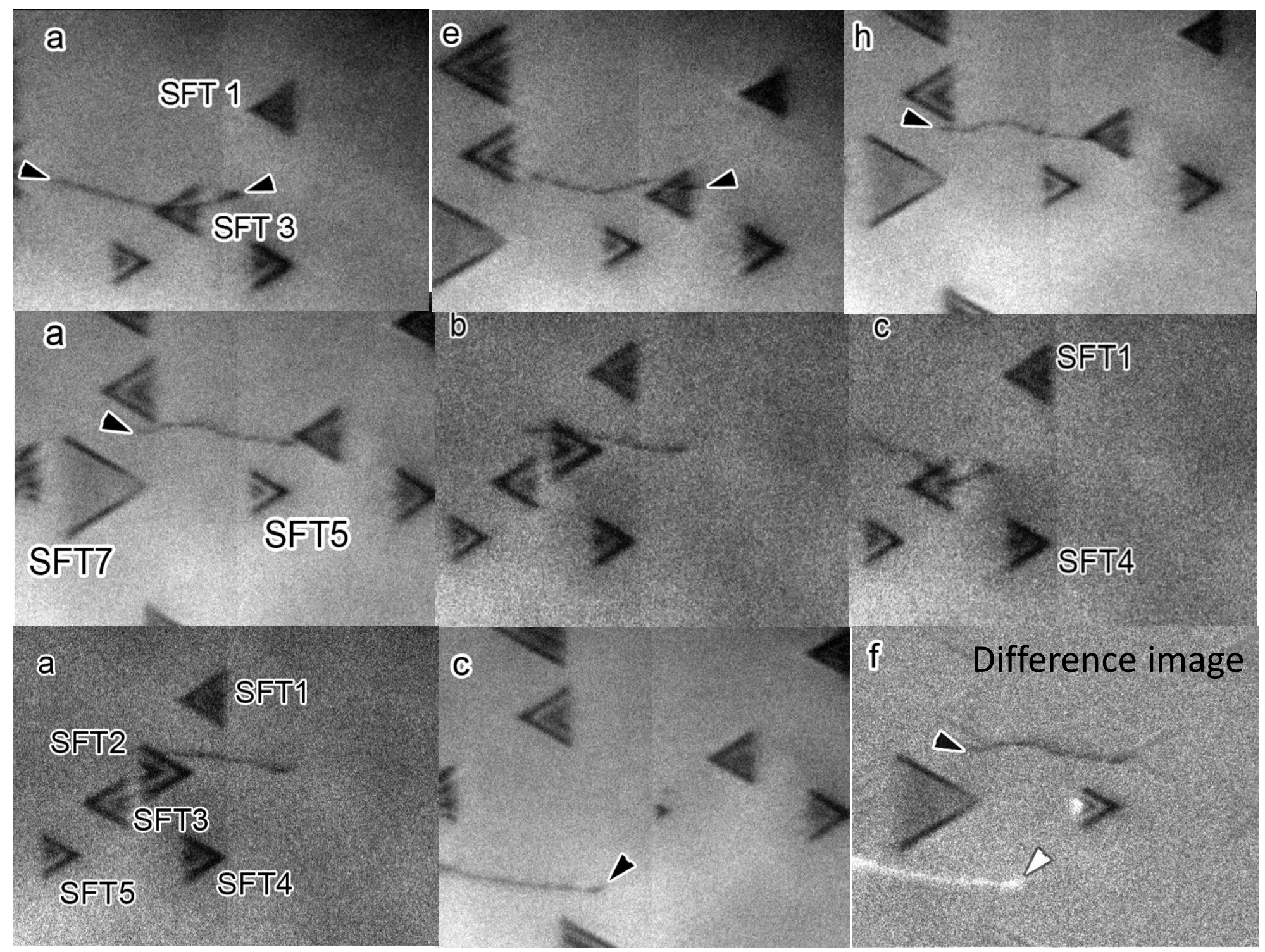

Pinning, magnitude unknown

Annihilation

Annihilation and replacement 


\section{Interaction of dislocations with a grain boundary in the ion irradiated austenitic stainless steel}

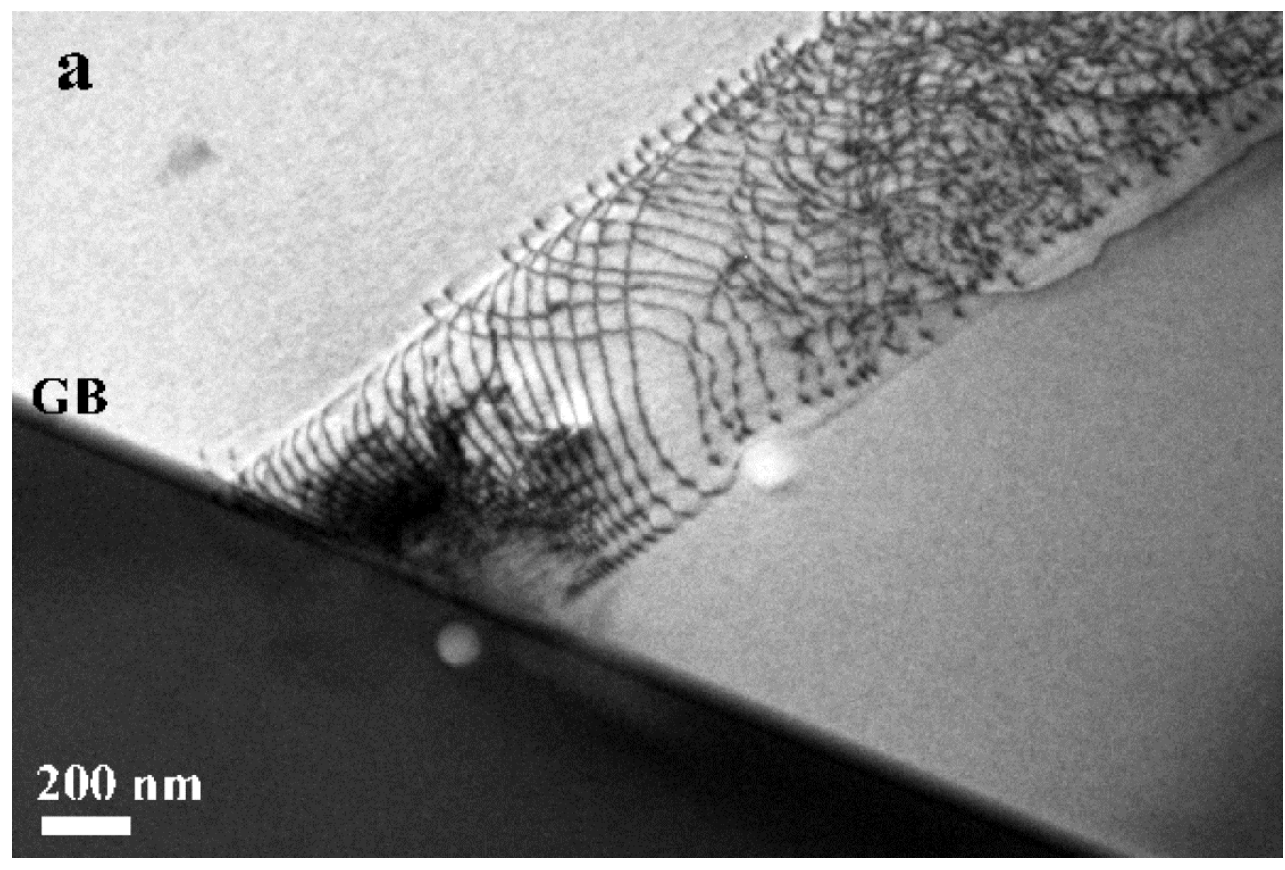

Two incoming systems produce four outgoing systems.

Are the outgoing systems predicted by the transfer rules predicted for unirradiated fcc metals?

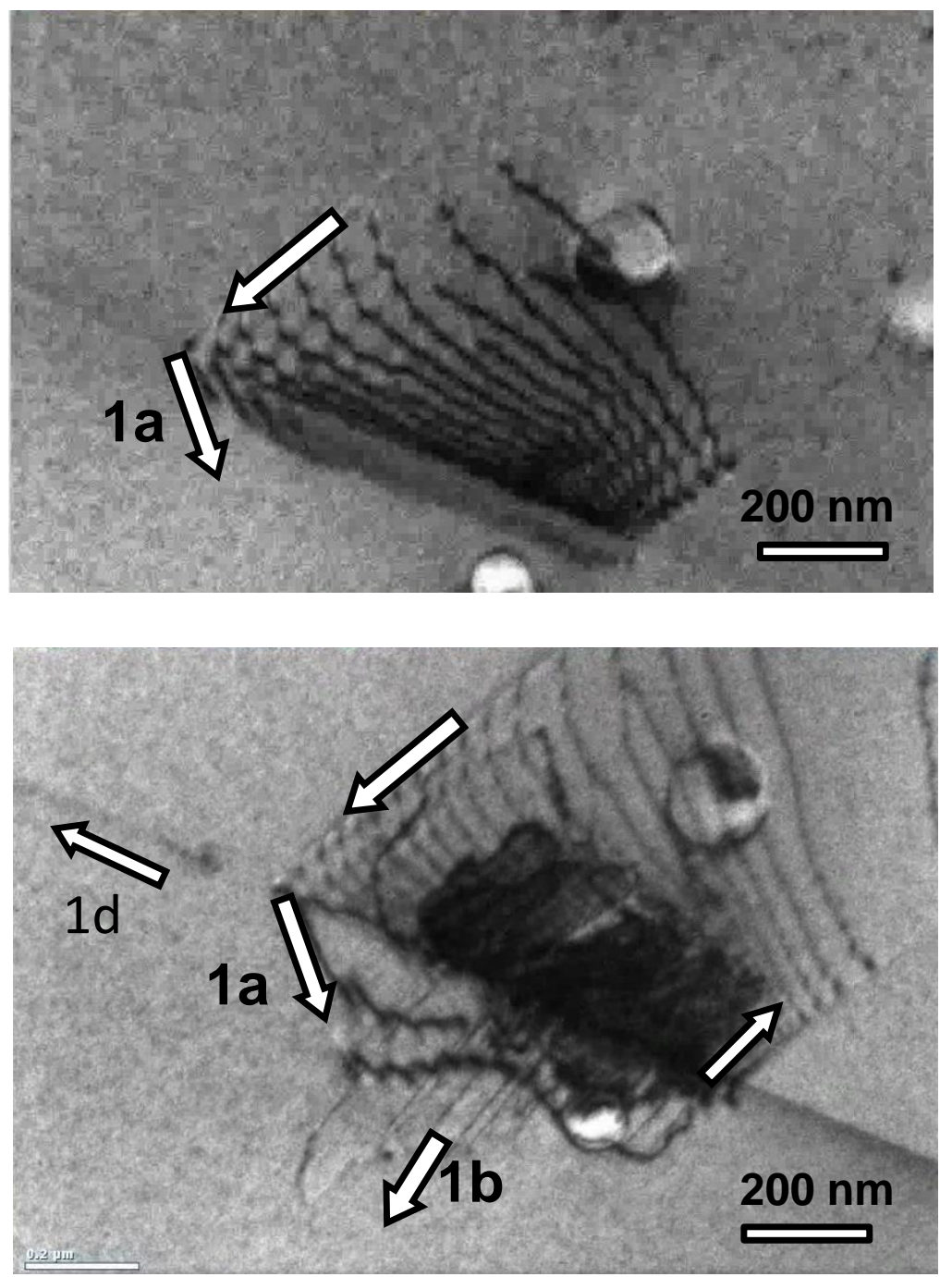




\section{Interaction of dislocations with grain boundaries in irradiated austenitic steels.}

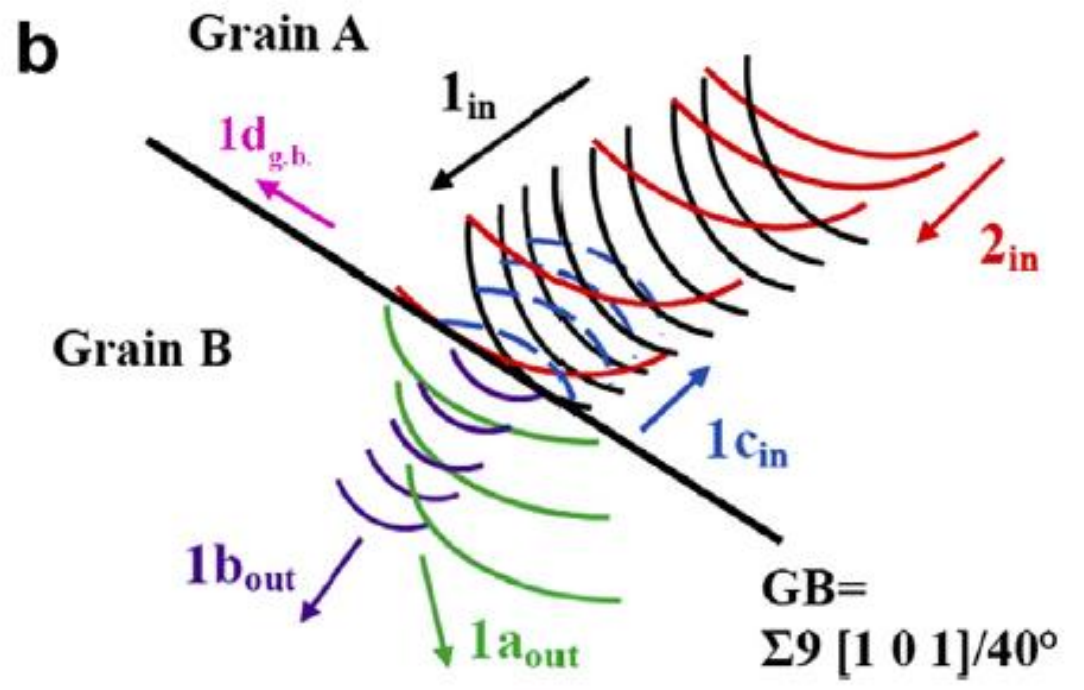

Conclusion: Slip transfer across a grain boundary is determined by the magnitude of the residual grain boundary dislocations generated by the transfer process but the magnitude of the resolved shear stress must be sufficient to propel the dislocations through the field of radiation damage.

Transition from nucleation stress to propagation stress dominance.
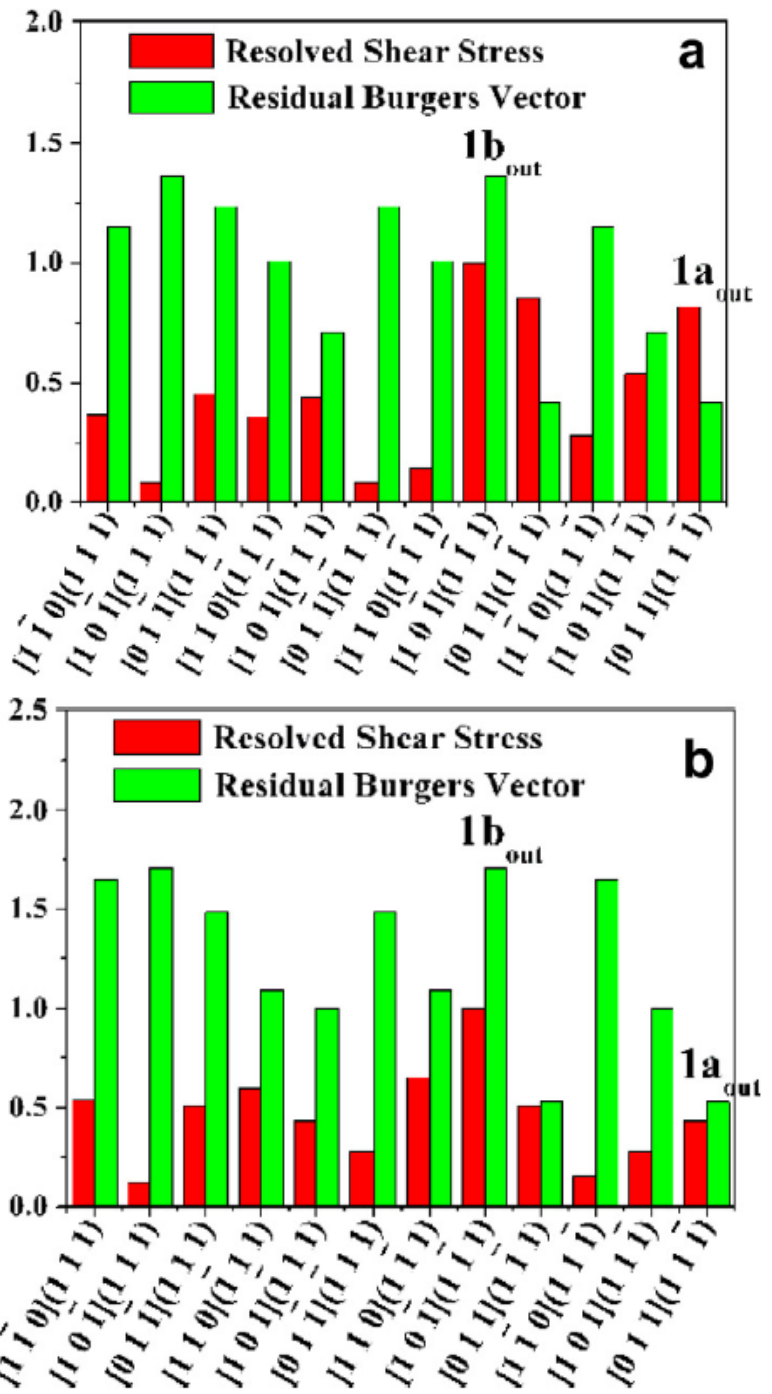


\section{Dislocation interactions with grain boundaries in irradiated steel}
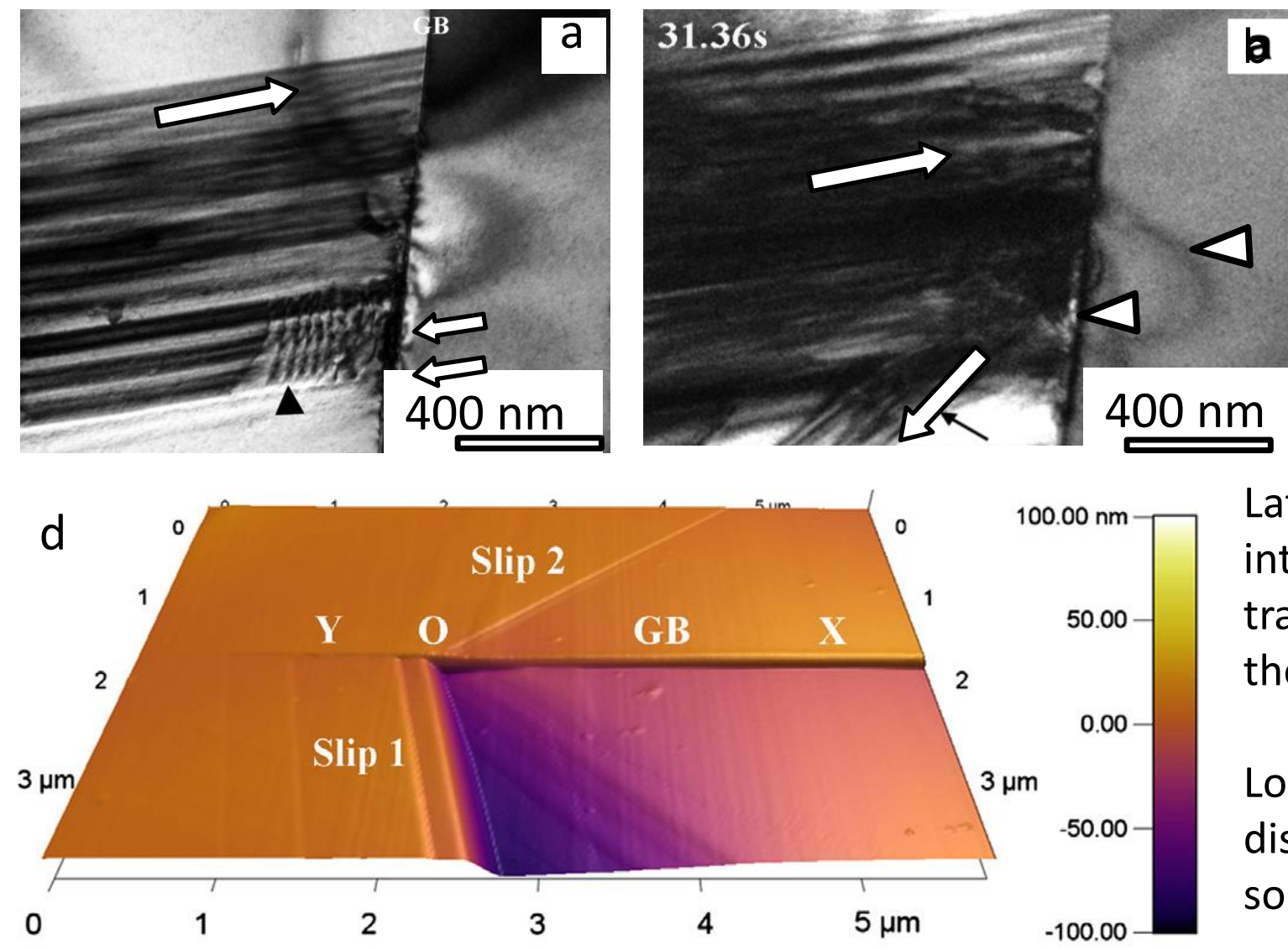

Cui B et al .Acta Mater. 2014;65:150. Support DOE DE-FG02-08ER46525

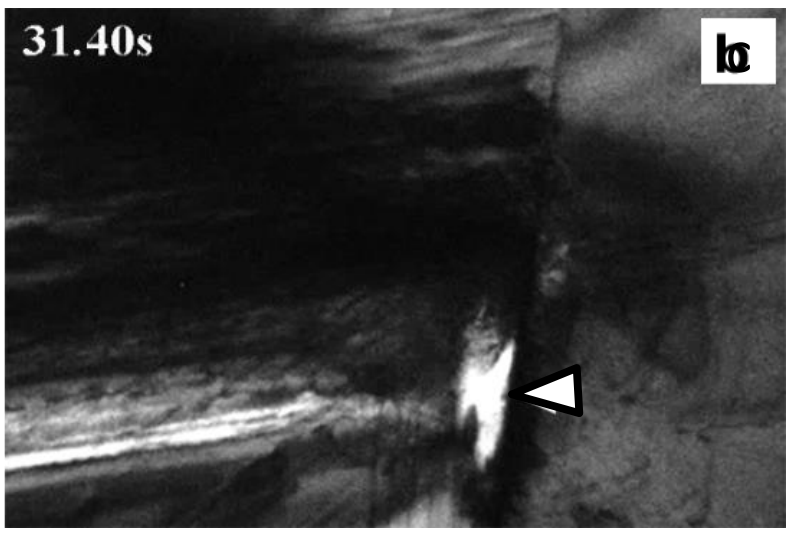

Lattice dislocation accommodation into the grain boundary. No transmission of slip but cracking of the grain boundary.

Local stress insufficient to propagate dislocations out from grain boundary sources.

Key finding: - process for the disruption of the protective oxide related to dislocation interactions with the grain boundary. 


\section{HREBSD Stress Distribution Comparison: Discontinuous vs Continuous Channels}
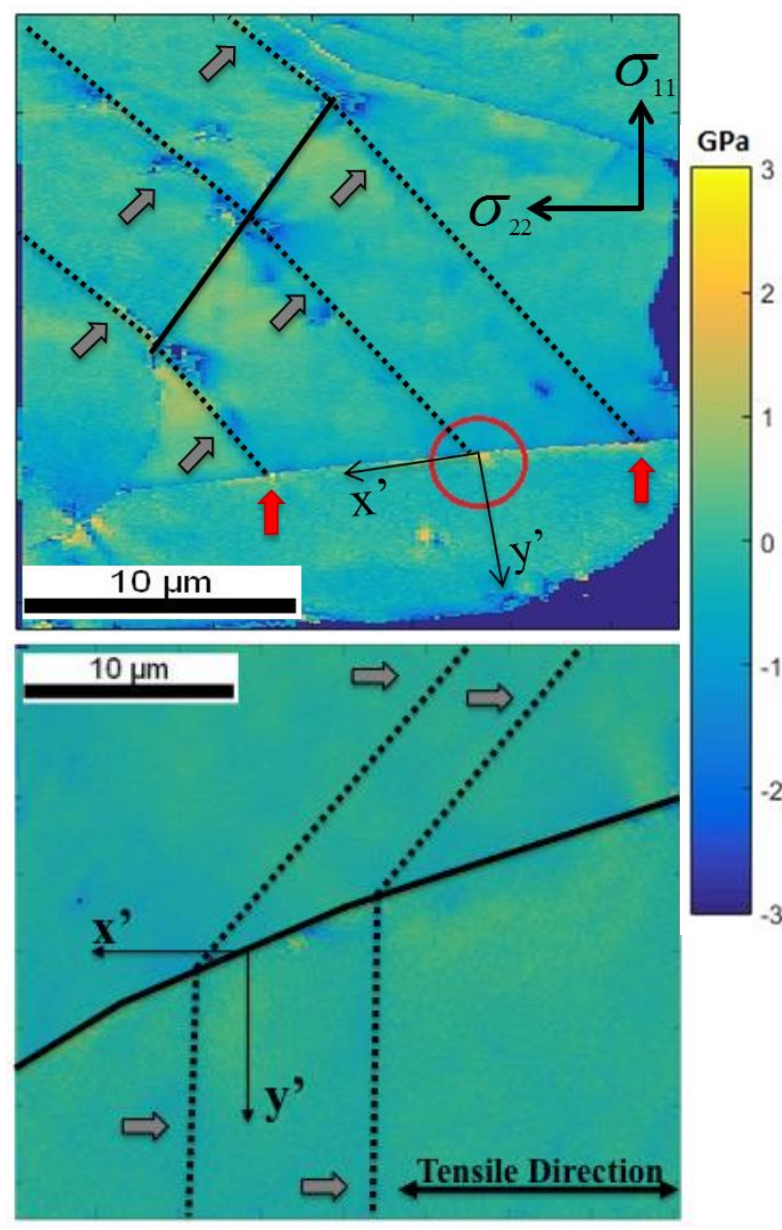
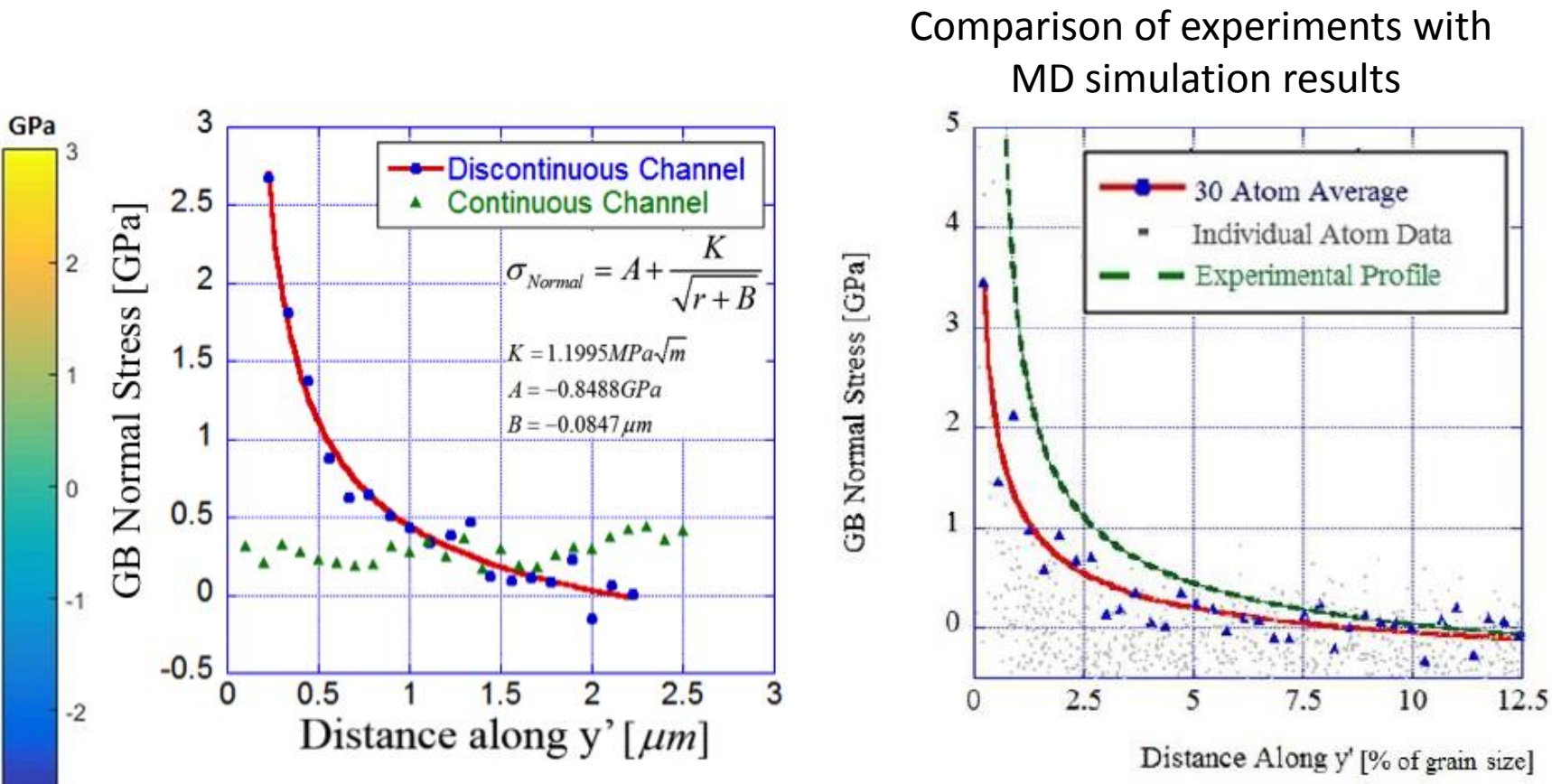

Is there a critical stress for nucleating and propagating dislocations from a grain boundary in an irradiated metal? 


\section{Summary and conclusions}

- The presence of an irradiation hardened matrix does not change the condition for the selection of the activated slip system to transfer strain across a grain boundary

- The presence of an irradiation hardened matrix transitions the rate limiting step from nucleation of dislocations from grain boundary sources to propagation away from the grain boundary.

GB: $\sum 39(111) / 32.2^{\circ}$ twist

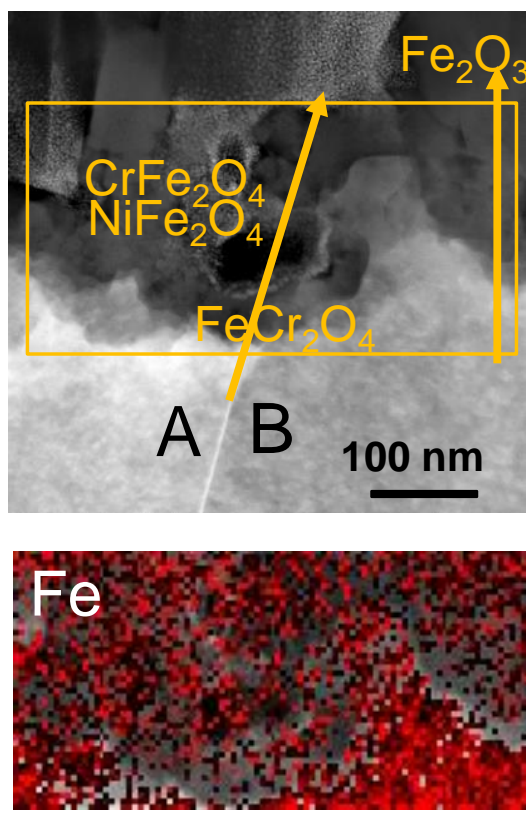

- Grain boundary source is not a line source but an area of the grain boundary. This impacts the width of the channels.

- Pre-existing dislocations essentially frozen in place by the irradiation.
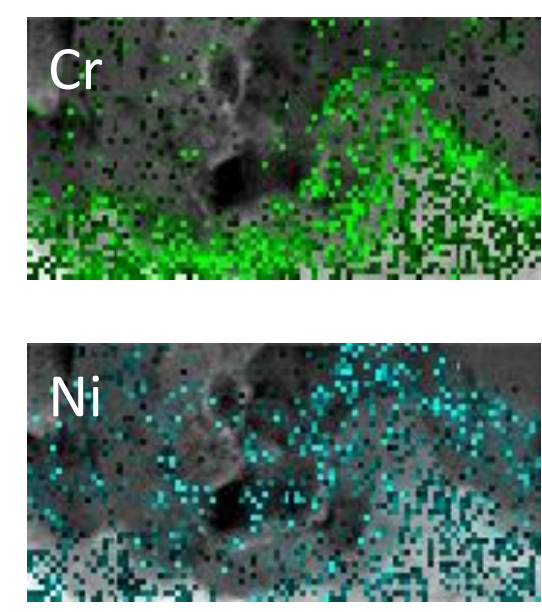

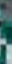

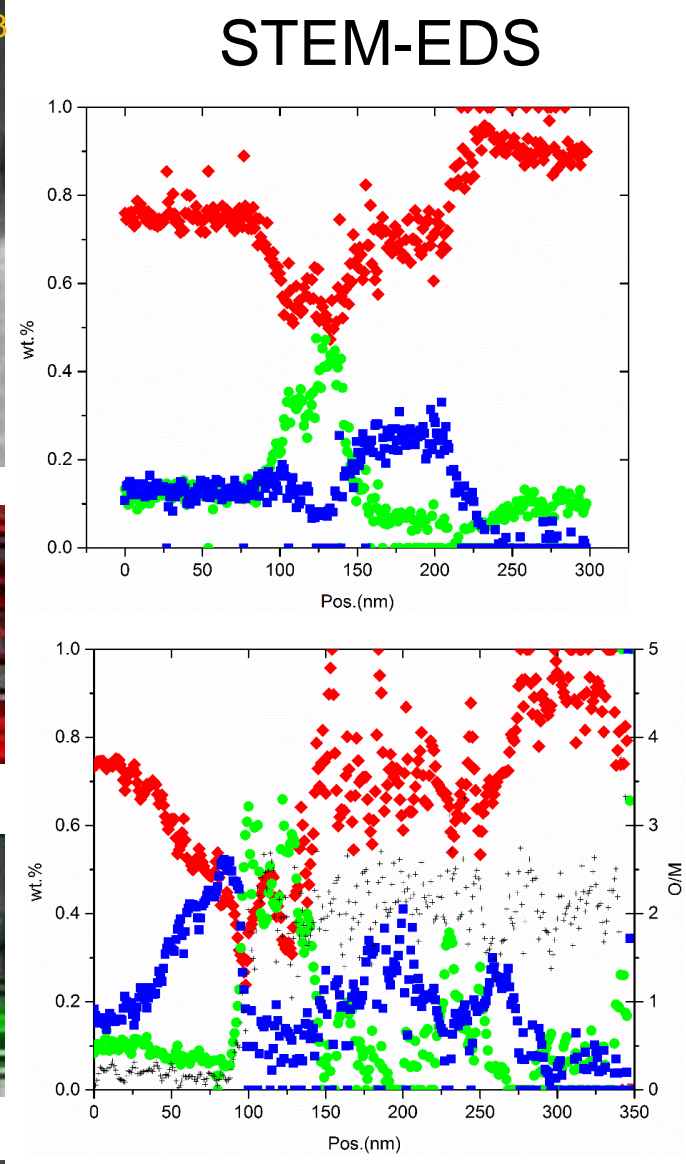

STEM-EDS 